\title{
Diversité et structure des espaces végétalisés urbains de la ville de Ziguinchor, Sénégal
}

\author{
Mohamed M. CHARAHABIL ${ }^{1 *}$, Bassène CESAR $^{2}$, Baldé HAMADOU ${ }^{3}$, \\ Seydou NDIAYE ${ }^{1}$ et Malaïny DIATTA ${ }^{4}$
}
${ }^{I}$ Laboratoire d'Agroforesterie et d'Écologie (LAFE) - Département Agroforesterie/UFR ST/, Université Assane Seck de Ziguinchor/Sénégal.
${ }^{2}$ Section Productions Végétales et Agronomie, UFR des Sciences Agronomiques, de l'Aquaculture et des Technologies Alimentaires. Université Gaston Berger de Saint Louis/Sénégal.
${ }^{3}$ Master's Student in Agricultural Structures and Irrigation at Ondokuz Mayls University/Turkey.
${ }^{4}$ Centre National de Recherche Forestière (CNRF/ISRA). Institut Sénégalais de Recherche Agricole (ISRA/Sénégal).
*Auteur correspondant ; E-mail: mcharahabil@univ-zig.sn; Tel : (+221) 775320107

\section{RÉSUME}

Dans les pays en développement les deux tiers de la croissance démographique sont absorbés par les villes. Ce phénomène entraine, et souvent de manière irréversible, une érosion de la biodiversité végétale dans les espaces urbains et périurbains. Ce travail, qui constitue une première contribution à l'étude des Espaces Végétalisés Urbains (EVU) de la ville de Ziguinchor, a pour objet de mettre en évidence la diversité et l'organisation du peuplement végétal dans cette ville à urbanisation galopante. Il a été réalisé à partir d'inventaires floristiques itinérants réalisés entre 2014 et 2016. Les résultats présentent la composition floristique des arbres de la ville, leur statut géographique et ainsi que leur organisation spatiale. La diversité floristique montre une richesse de 132 espèces réunies dans 95 genres et 32 familles. Les Quatre (4) familles les plus dominantes sont les Fabaceae (19 espèces), les Moraceae (18 espèces), les Euphorbiaceae (13 espèces) et les Apocynaceae (11 espèces). Les cinq (5) espèces les plus fréquentes sont Azadirachta indica avec une fréquence relative de $90 \%$ suivie de Mangifera indica (69\%), d'Elaeis guineensis (55\%), de Adansonia digitata (52\%) et Borassus aethiopum (45\%). La typologie des habitats et de la végétation réalisée à partir d'une Analyse Factorielle des Correspondances (AFC) a mis en évidence des zones sacrées pour les populations urbaines tels que les cimetières et les bois sacrés qui conservent des espèces rares et menacées tels Cassia sieberiana, Ficus basarensis, Ficus ottoniaefolia, Ficus ovata et Ficus platyphyla. Comparée à la flore des autres villes africaines, la ville de Ziguinchor conserve encore une diversité végétale originale qui mérite plus de regard pour les biens et services non négligeables qu'elle peut apporter aux populations urbaines et périurbaines.

(C) 2018 International Formulae Group. All rights reserved.

Mots clés: flore, typologie, urbanisation, zone sacrée, Ziguinchor, Casamance. 


\title{
Diversity and structure of urban plant areas of the town of Ziguinchor, Senegal
}

\begin{abstract}
In developing countries two-thirds of population growth is absorbed by cities. This phenomenon leads, and often irreversibly, to an erosion of plant biodiversity in urban and peri-urban areas. This work, which is a first contribution to the study of Urban Vegetated Spaces (EVU) of the city of Ziguinchor, aims to highlight the diversity and organization of plant populations in this city with galloping urbanization. It was carried out between 2014 and 2016 through itinerant floristic inventories. The results present the floristic composition of the trees in city, their geographical status and as well as their spatial distribution. The floristic diversity is rich of 132 species distributed in 95 genera and 32 families. The four (4) most dominant families are Fabaceae (19 species), Moraceae (18 species), Euphorbiaceae (13 species) and Apocynaceae (11 species). The five (5) most common species are Azadirachta indica with a relative frequency of $90 \%$ followed by Mangifera indica (69\%), Elaeis guineensis (55\%), Adansonia digitata (52\%) and Borassus aethiopum (45\%). ). The typology of habitats and vegetation based on a Correspondence Factor Analysis (AFC) has highlighted sacred areas for urban populations such as cemeteries and sacred groves that conserve rare and endangered species such as Cassia sieberiana, Ficus basarensis, Ficus ottoniaefolia, Ficus ovata and Ficus platyphyla. Compared with the flora of other African cities, the city of Ziguinchor still retains an original plant diversity that deserves more attention for the significant goods and services it can bring to urban and peri-urban populations.
\end{abstract}

(C) 2018 International Formulae Group. All rights reserved.

Keywords: flora, typology, urbanization, sacred areas, Ziguinchor, Casamance.

\section{INTRODUCTION}

De nos jours, les villes sont des espaces fortement peuplés et font face à l'urbanisation graduelle qui est le résultat des effets de la croissance démographique sans précédent dans le monde en général et particulièrement en Afrique. En même temps, face à la recherche de solutions aux répercussions des changements climatiques en ville, une forte demande sociale, économique et environnementale ne cesse de croitre autour de l'évidence de la mise en place des espaces de nature en ville, arguant de ses avantages sanitaires, sociaux, environnementaux, et voire économiques qui permettraient d'asseoir des villes plus pérennes et attractives. C'est en ce sens que Tavin et Leseur (2016) soutiennent que ces espaces verts, privés et publics (parcs urbains, jardins privés, toits et murs végétalisés, linéaires arborés, friches et espaces vacants...), font en effet bénéficier la ville et ses habitants d'une multiplicité de services dits services écosystémiques (séquestration du carbone, régulation thermique, services de support de la biodiversité et des écosystémiques, d'approvisionnement et de purification de l'eau, espaces de loisirs, valorisation économique du foncier, purification de l'air, production via l'agroforesterie urbaine, etc.). Il faut ajouter, à ces bienfaits $d u$ végétal en ville, le renforcement de la cohésion sociale (Arnberger et Eder, 2012); la contribution directe à la santé des habitants en réduisant le stress, en favorisant l'activité physique, en améliorant le cadre de vie et l'état de santé ressenti (Dron et al., 2012 ; Kaczynski et al., 2007 cité par Laïlle et al., 2013).

Reconnaissant cette diversité de fonction des espaces végétalisés urbains, l'inquiétude des scientifiques pour la création des pôles urbains socialement viables, économiquement vivables et écologiquement durables et attractifs, en se basant sur l'étude de leur biodiversité végétale et leur service écosystémiques rendus, se conjugue et converge vers un intérêt commun à savoir la préservation des espaces végétalisés urbains. En revanche, plusieurs études mettant en exergue la biodiversité du végétal en ville existent dans le monde (Savard J.P., 2000 ; Cornelis et al., 2004 ; Saümel et al., 2010 ; Arnould et al., 2011; Vroh et al., 2014 ; Amontcha et al., 2015; Nomel et al., 2017 ; 
Dasylva et al., 2017). Cependant, une telle étude mettant en relief l'importance de la biodiversité végétale en ville au Sénégal reste rare, voire inexistante.

La flore du Sénégal, l'une des mieux étudiées de la région ouest-africaine est estimée à 3589 espèces (Ba et al., 2001) dont 2457 (98,3\%) appartiennent au groupe des Angiospermes constitué de 997 genres et 162 familles. La région de Ziguinchor regorge la plus grande part de cette diversité végétale, totalisant à elle seule environs 1150 espèces végétales répertoriées (Sané et al., 2017).

Les résultats issus de ces recherches ont attiré l'attention des autorités, des chercheurs et des touristes. C'est ainsi qu'a vu le jour plusieurs initiatives de conservation in situ et ex situ de la biodiversité végétale telle que l'implantation du Centre de Formation Professionnelle des Eaux et Forêts (CFPEF) en 1963 à Djibélor. Ce centre est devenu plus tard le Centre National de Formation des Techniciens des Eaux, Forêts, Chasses et des Parcs Nationaux (CNFTEFCPN) dont l'objectif était de former des agents techniques supérieurs aptes à conserver les forêts. Par la suite, un arborétum destiné à la formation pratique des agents techniques des eaux et forêts et également de fournir une collection de certaines espèces végétales importantes de la zone a été mis en place. En outre, les prospections botaniques réalisées dans les forêts classées ayant une superficie estimée à 116776,30 ha (ANSD, 2011), ont enrichi de 2000 nouvelles espèces endogènes et environ 300 espèces introduites (Ba et al., 2001) dans la $2^{\mathrm{e}}$ édition de la flore du Sénégal.

Malheureusement, après cette période, la flore du Sénégal et celle de la région de Ziguinchor en particulier sont très peu révisées. Dans la littérature, on ne retrouve que les travaux de (Vanden Berghen et Diasssy (2001) ; Vanden Berghen (1982, 1984, 1985), Doumbia (1966) et Compère (1991) portant sur les plantes aquatiques.

Il n'est plus à démontrer que l'urbanisation, les nouvelles pratiques culturales et forestières et changements climatiques sont les principales causes de la perte de biodiversité, de la modification ou perte d'habitats et de la diminution des services écosystémiques rendus à l'homme. Cela est d'autant plus vrai en Afrique où la dynamique de transformations des villes et villages est importante et le besoin des populations vis-àvis des plantes est plus marqué (Botolisam et al. 2014). Par ailleurs, dans la ville de Ziguinchor, la diminution des espaces verts s'explique particulièrement par l'expansion spatiale de la ville qui se heurte à des contraintes physico-géographiques très marquées (au Nord une cuvette «étranglée» par le fleuve Casamance, à l'Est et l'Ouest les marigots de Boutoute et de Djibélor, respectivement).

Aux vues de cet état de fait, il est devenu urgent de sensibiliser l'ensemble des citoyens à l'importance de la biodiversité locale et l'urgence de la préserver. Cette étude se veut être un point de départ d'un grand nombre de recherches sur les espaces végétalisés de la ville de Ziguinchor et permettra d'assoir les bases essentielles de la connaissance des ressources naturelles, pour la mise en valeur de cette localité.

\section{MATERIEL ET METHODES \\ Présentation de la zone d'étude Cadre biophysique}

La présente étude a été réalisée au sud du Sénégal entre 2014 et 2016, dans la commune de Ziguinchor. Cette commune de Ziguinchor, chef-lieu de région du même nom est située entre la latitude $\mathrm{d}^{\circ} \mathrm{d}^{\prime} \mathrm{d} "$ et $13^{\circ}$ et la longitude $16^{\circ}$ et $d^{\circ} d^{\prime} d^{\prime \prime}$. Elle est limitée au Nord par le fleuve Casamance, au Sud, à l'Est et à l'Ouest par la communauté rurale de Niaguis (Figure 1).

\section{Climat}

Située dans le domaine soudanoguinéen, la région de Ziguinchor a un climat chaud et humide, avec une température moyenne de $27{ }^{\circ} \mathrm{C}$. Ainsi, on note une longue saison sèche qui dure environ sept à huit mois (novembre-mai) et une courte saison des pluies généralement abondante qui dure environ quatre à cinq mois (Juin-Octobre). La Basse Casamance constitue la partie la plus arrosée du Sénégal en dépit d'une pluviométrie irrégulièrement répartie dans le temps et dans l'espace avec une moyenne annuelle de 
$1253,83 \mathrm{~mm}$ pendant les trois dernières décennies (Figure 2).

\section{Flore}

La région de Ziguinchor est la plus diversifiée du pays en ressources végétales avec 1150 espèces végétales sur les 2500 espèces répertoriées (MEPN, 1997). La forêt demi-sèche dense à deux étages, rencontrée dans la région est caractéristique du domaine guinéen. Elle est caractérisée par des espèces telles que khaya senegalensis (Caïcédrat), Parinari excelsa (Mampatan), Ceiba pentandra (Fromager), Chrolophora regia (Iroko), Antiaris africana (Tomboiro), Detarium senegalense (Ditakh), Erythrophleum guineense (Tali), Afzelia africana (Link) et Elaeis guineensis (Palmier à huile). Dans cette forêt, le sous-bois est formé d'arbrisseaux sarmenteux, d'herbes et de lianes dont les espèces Saba senegalensis et Landolphia heudelotii qui jouent un rôle important dans l'économie des ménages. La mangrove, que l'on rencontre dans la région, est dominée par les palétuviers du genre Rhizophora et Avicennia (Badiane, 1984 cité par Montoroi, 1993).

\section{Faune}

Le parc national de la basse Casamance, situé dans la région, est très diversifié en ressources fauniques. On y trouve plus de cinquante espèces de mammifères dont quelques espèces devenues rares comme Pantherus pardus (Panthère), Syncerus caffer nanus (Buffle de forêt), Colobus badius (Colobe bai), Trichechus senegalensis (Lamantin), Orycteropus afer (Oryctérope) (MEF \& DCEF, 2004).

L'ichtyofaune de la Casamance compte 75 espèces réparties en 18 familles. On rencontre aussi une quarantaine d'espèces marines, une trentaine d'espèces estuariennes et quelques-unes continentales (Dasylva, 2013). Les reptiles sont représentés par Crocodilus niloticus (Crocodile du Nil), Varanus niloticus (Varan du Nil), Python sebae (Python) etc.
Sols

Les sols rencontrés dans la région de Ziguinchor sont:

$>$ Les sols salés sulfatés acides et hydromorphes ou peu évolués dans les vallées ;

$>$ Les sols faiblement ferrallitiques sur les plateaux et formations géo-argileux, kaolinisées profondes (CSE, 2012).

\section{Cadre socio-économique}

Le recensement général de la Population et de l'Habitat, de l'Agriculture et de l'Élevage (RGPHAE) de 2013 (ANSD, 2015) précise que 549151 personnes habitent la région de Ziguinchor (4\% de la population du Sénégal). La densité de population est de 75 habitants au $\mathrm{km}^{2}$. Celle de la commune estimée par le MCA-S en 2013 est de 276354 habitants alors qu'en 2010 le taux d'urbanisation était de $47,15 \%$. La région est riche d'une grande diversité ethnique et culturelle. Les principales ethnies sont les Diolas 57,80\%, les Mandingues $11,10 \%$, les Peuls $10,50 \%$, les wolofs 3,90\%, les Manjacks 3,50\%, les Ballantes 2,90\%, les Sérères $2,70 \%$ et les Mancagnes 2,40\% (MCA-S, 2013). Les religions dominantes sont l'Islam (78\%) et le Christianisme (18\%), néanmoins, on trouve une forte communauté d'animistes et de païens dans le département d'Oussouye $(32,70 \%)$ (MCA-S, 2013). L'économie de la région est largement dominée par le secteur primaire qui occupe les $90 \%$ des actifs durant 3 à 4 mois dans l'année (MCA-S, 2013). Ce secteur comprend principalement l'agriculture essentiellement hivernale et tributaire des aléas climatiques, l'exploitation forestière, la pêche et l'élevage. Il s'y ajoute l'artisanat et le commerce. Les différents types de cultures pratiqués dans la région sont: les cultures céréalières, les cultures de rentes et les cultures maraîchères.

\section{Méthodes d'étude \\ Inventaires floristiques}

La méthode d'inventaire utilisé est la méthode itinérante. Elle consiste à réaliser des relevés phytoécologiques au niveau des points verts de la commune de Ziguinchor (Figure 1). 
À l'aide de Google Earth, les sites de hautes végétations ont été superposés sur une carte de stratification de la commune de Ziguinchor. Ce repérage a permis d'identifier 13 sites dans lesquels 29 relevés ont été réalisés. L'établissement de la liste des espèces recensées est fait par un inventaire itinérant qui consiste à inventorier toutes les espèces en parcourant une surface d'observation, définie en fonction de l'hétérogénéité des milieux, dans différentes directions et en notant le nom de l'espèce nouvellement rencontrée (Noba, 2002).

Les identifications ont été réalisées à l'aide de la flore illustrée du Sénégal (Berhaut, 1971, 19751979 et 1979), et (Mougnier, 2008), des ligneux du sahel (Arbonier, 1982). La nomenclature utilisée est celle de Lebrun et
Stork (1991, 1992, 1995, 1997). La classification APG III 2009 est celle utilisée pour la nomenclature des taxas.

La détermination des origines des espèces (endogènes ou exogènes) a été établie à l'aide de ces mêmes documents.

\section{Traitement des données}

La fréquence relative d'une espèce a été calculée en établissant le rapport entre le nombre de relevés où l'espèce est présente et le nombre total de relevés. La typologie des habitats de la végétation a été appréhendée à l'aide d'une Analyse Factorielle des Correspondances (Figure 3).

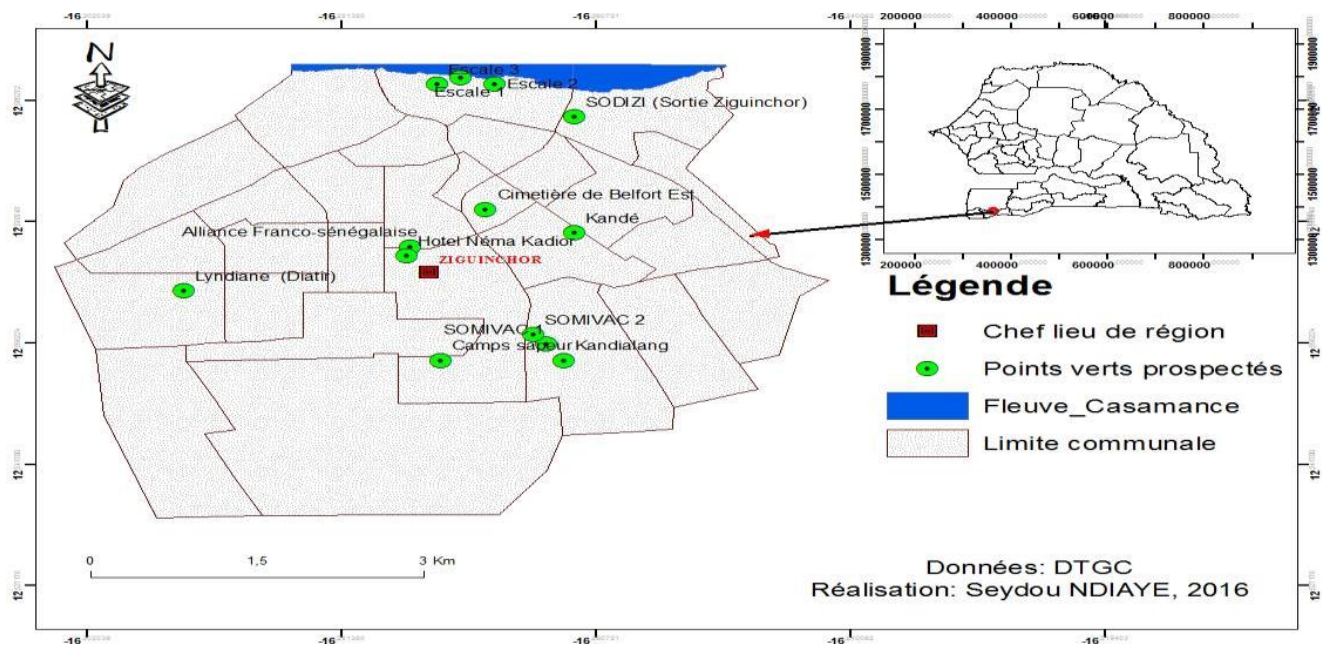

Figure 1 : Localisation des points verts prospectés de la commune de Ziguinchor.

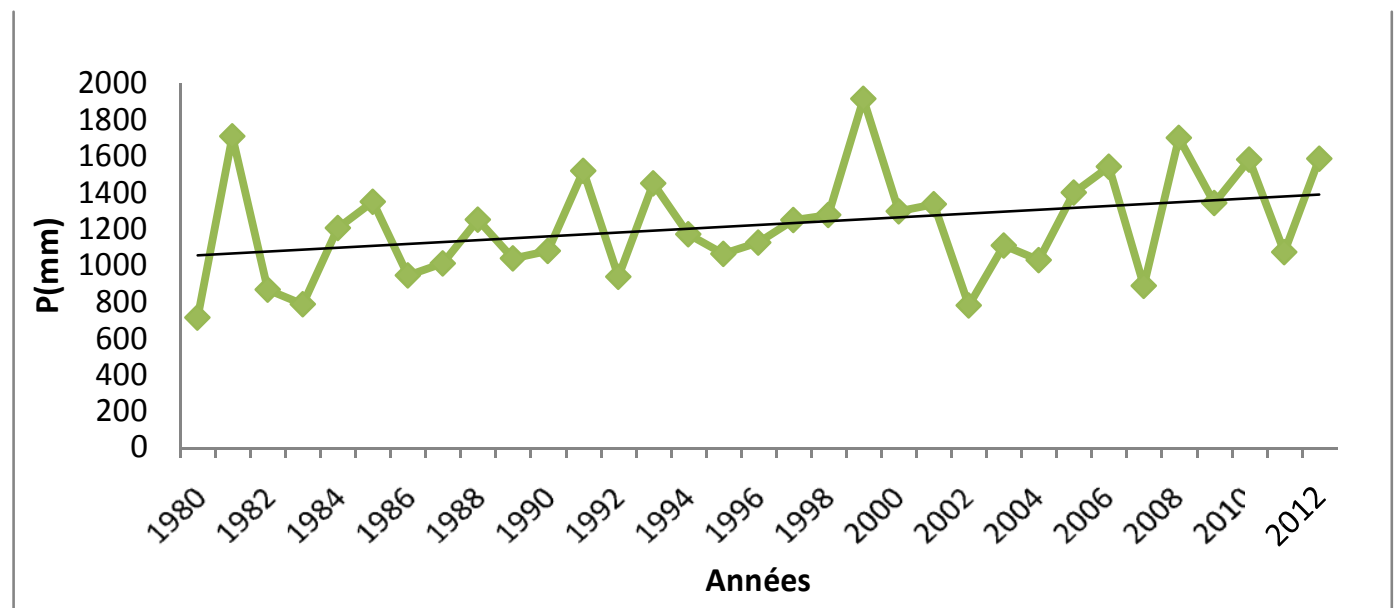

Figure 2 : Variation de la pluviométrie de la commune de Ziguinchor entre 1980 et 2012. 
(axes F1 et F2 : 18,64 \%)
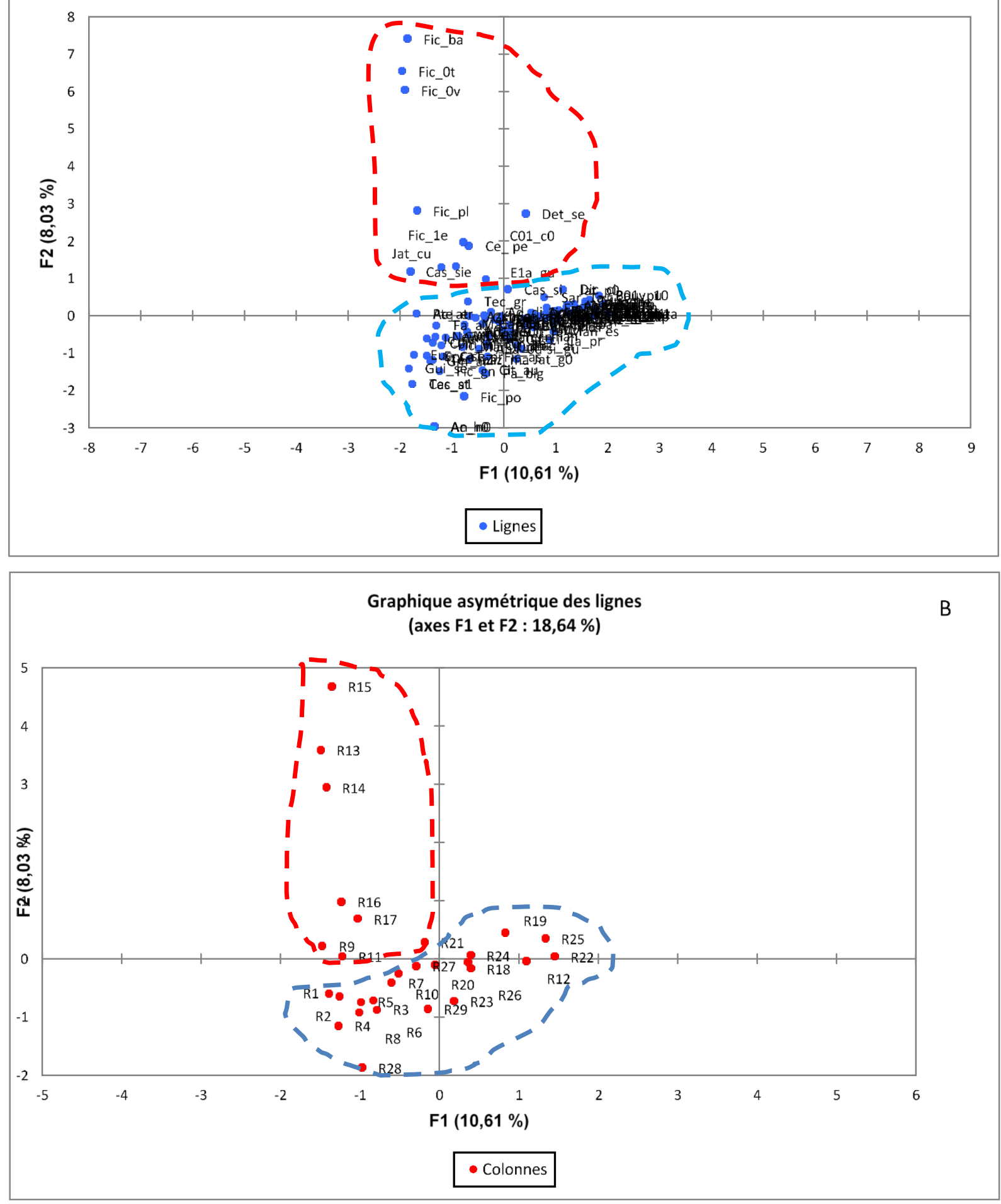

Figure 3 : Cartes de 1'AFC sur le plan des axes F1\&F2 de la matrice 132 espèces/29 relevés A (espèces), B (relevés). 


\section{RÉSULTATS \\ Étude de la flore}

La flore de la ville est uniquement constituée de Spermaphytes (Tableau 1). Le sous-embranchement des Gymnospermes est représenté par une seule famille (Cycadaceae), le reste est constitué des Angiospermes. L'analyse de l'origine des espèces (endogènes ou exotiques) montre que $53 \%$ des arbres et arbustes de la ville sont introduits contre $47 \%$ d'espèces endogènes.

\section{Spectre taxonomique}

L'analyse du spectre taxonomique montre que la flore ligneuse de la commune de Ziguinchor est riche de 132 espèces réunies dans 95 genres et 32 familles. Dans cette flore, les Angiospermes représentent 97,89\% des espèces recensées contre $2,11 \%$ de Gymnospermes. Pour les Angiospermes, les Dicotylédones représentent $86,36 \%$ contre 5,3\% de Monocotylédones (Tableau 2).

L'importance relative des familles est consignée dans le Tableau 3. Quatre (4) familles sont dominantes avec un nombre d'espèces supérieures à 10 . Ces principales familles représentent près de la moitié des espèces listées soit $46,21 \%$ et appartiennent à la classe des Dicotylédones. Il s'agit de la grande famille Fabaceae (19 espèces), des Moraceae (18 espèces), des Euphorbiaceae (13 espèces) et des Apocynaceae (11espèces). Les $53,79 \%$ restant sont constitués des familles qui présentent moins de 10 espèces.

Dans ce groupe, nous avons 3 familles qui sont représentées chacune par 6 espèces, 2 familles représentées chacune par 5 espèces, 2 familles représentées chacune par 4 espèces, 4 familles représentées chacune par 3 espèces, 6 familles représentées chacune par 2 espèces et 11 familles représentées chacune par une (1) espèce.

\section{Fréquences de présence des espèces}

L'analyse de la distribution spatiale des espèces montre que 4 espèces sont les plus fréquentes. Elles sont présentes sur plus de la moitié des relevés réalisés. Il s'agit respectivement de 2 espèces exogènes (Azadirachta indica avec une fréquence relative de $90 \%$ et Mangifera indica [69\%]) et 2 espèces endogènes (Elaeis guineensis [0, 55\%] et Adansonia digitata [0, 52\%]).

\section{Étude de la végétation}

La typologie des habitats et de la végétation a été établie à l'aide d'une analyse factorielle de correspondance réalisée sur la matrice de 29 relevés/132 espèces. La contribution des espèces a varié de 0,01 à $8,3 \%$ et celle des relevés de 0,02 à $23,2 \%$. La contribution moyenne des relevés est estimée à $34 \%$ l'information contenue dans le tableau, contre $8 \%$ pour les espèces. Les espèces et les relevés à forte contribution autrement dit présentant une valeur supérieure à la moyenne sur l'ensemble des deux axes (F1/F2) sont respectivement

- $\quad$ Ficus ottoniaefolia (8,3\%), Ficus ovata (7,1\%), Ficus platyphyla (5,7\%), Ficus basarensis $(5,2 \%), \quad$ Ceiba pentandra (3,5\%), Cola cordifolia (3,2\%), et Detarium senegalense (2\%) pour les espèces.

- $\quad$ R 15 (23,2\%), R 22 (12,68\%), R13 $(8,06 \%), \mathrm{R} 14$ (7,62\%), R4 (5,9\%), R25 $(5,55 \%)$ et R28 $(4,4 \%)$.

Sur la base de ce groupe d'espèces et de relevés significatifs, on peut dresser l'organisation des espaces végétalisés de la ville.

On retrouve en effet dans l'extrémité positive de l'axe F1 les relevés effectués dans les jardins publics comme à l'alliance (R22) et dans les domiciles particuliers situés en ville (R25). Ces relevés sont associés à des espèces généralement exotiques (Azadirachta indica, Bauhinia reticulata, Bougainvillea glabra, Bougainvillea spectabilis , Cassia siamea, Ceiba pentadra, Cocos nucifera, Cola cordifolia ; Cordia sinensis, Khaya senegalensis, Mangifera indica, ...). À l'opposée, on retrouve les relevés effectués dans les espaces végétalisés spontanés et moins affectés par l'action de l'homme. Il s'agit du cimetière et de ces environs ( $\mathrm{R} 15, \mathrm{R} 13, \mathrm{R} 14)$, de petites forêts intactes que l'on retrouve dans le camp des sapeurs-pompiers par exemple et dans la zone sud de la ville (Lyndiane-Diatir). 
Les espèces qui $\mathrm{y}$ sont inféodées sont essentiellement endogènes et d'autres sont particulièrement (Ceiba pentandra, Cola cordifolia, Elaeis guineensis, Faidherbia albida, Ficus ovata, Ficus platyphyla, Ficus basarensis, Ficus ottoniaefolia, Ficus ovata, Ficus platyphyla).
L'axe F2 permet de distinguer dans son extrémité positive un groupe de 10 espèces toutes endogènes dont cinq du genre Ficus $(F$. basarensis, $F$. ovata, $F$. ottoniaefolia, $F$. platyphyla, $F$. leucardii), D. senegalensis, $C$. cordifolia, C. pentadra, C. sieberiana, E guinneensis.

Tableau 1 : Liste des espèces ligneuses inventoriées dans la ville de Ziguinchor par ordre alphabétique des familles.

\begin{tabular}{|c|c|c|c|c|}
\hline Famille & ; & Espèces & $\mathbf{F r}$ & Ind. /Exo \\
\hline \multirow[t]{2}{*}{ Agavaceae (D) } & & Dracaena arborea (Wild.) Link. & 0.14 & Exo \\
\hline & & Furcraea foetida (L.) HAW & 0.07 & Exo \\
\hline \multirow[t]{2}{*}{ Anacardiaceae (D) } & & Anacardium occidentale $\mathrm{L}$. & 0,31 & Exo \\
\hline & & Mangifera indica $\mathrm{L}$. & 0,69 & Exo \\
\hline \multirow[t]{3}{*}{ Annonaceae (D) } & & Annona muricata $\mathrm{L}$. & 0,17 & Exo \\
\hline & & Annona squamosa $\mathrm{L}$. & 0,07 & Exo \\
\hline & & Polyalthia longifolia Sonn. & 0,03 & Exo \\
\hline \multirow[t]{11}{*}{ Apocynaceae (D) } & \multirow[t]{2}{*}{ Asclepioidae } & Calotropis procera Ait. & 0,24 & Ind \\
\hline & & Leptadenia hastata (Pers. ) Decne. & 0,07 & Ind \\
\hline & \multirow[t]{9}{*}{ Apocynioidae } & Allamanda blanchetiiA.DC. & 0.03 & Exo \\
\hline & & Allamanda cathartica $\mathrm{L}$. & 0,17 & Exo \\
\hline & & Alstonia boonei De Wild. & 0.14 & Ind \\
\hline & & Holarrhena floribunda (G. Don.) Dur. & 0,14 & Ind \\
\hline & & Landolphia heudelotii A.DC. & 0.03 & Ind \\
\hline & & Nerium oleander L. & 0.14 & Exo \\
\hline & & Plumeria rubra $\mathrm{L}$. & 0.24 & Exo \\
\hline & & Saba senegalensis (A. DC.) Pichon. & 0,17 & Ind \\
\hline & & Thevetia neriifolia Juss. & 0,03 & Exo \\
\hline \multirow[t]{4}{*}{ Araliaceae (D) } & & Aralia filicifolia Chr.Moore. & 0,07 & Exo \\
\hline & & Panax fruticosum L.var.victoriae & 0,10 & Exo \\
\hline & & Polyscias guilfoylei var. laciniata Bailey. & 0.17 & Exo \\
\hline & & Polyscias scutellaria (Burm.f.) Fosberg. & 0.10 & Exo \\
\hline \multirow[t]{5}{*}{ Arecaceae (M) } & & Areca catechu L. & 0.14 & Exo \\
\hline & & Borassus aethiopum Mart. & 0,45 & Ind \\
\hline & & Cocos nucifera $\mathrm{L}$. & 0,31 & Exo \\
\hline & & Elaeis guineensis Jacq. & 0.55 & Ind \\
\hline & & Hyphaene thebaïca Mart. & 0,03 & Ind \\
\hline
\end{tabular}




\begin{tabular}{|c|c|c|c|}
\hline & Phoenix reclinata Jacq. & 0,10 & Ind \\
\hline \multirow[t]{4}{*}{ Bignonaceae (D) } & Crescentia cujete L. & 0,14 & Exo \\
\hline & Markhamia tomentosa (Benth.) K. Sch. & 0.21 & Ind \\
\hline & Newbouldia laevis (P. Beauv.) Seem. & 0.28 & Ind \\
\hline & Tecoma stans (L.) H.B.K. & 0.03 & Exo \\
\hline \multirow[t]{3}{*}{ Boraginaceae (D) } & Cordia africana Lam. & 0,14 & Ind \\
\hline & Cordia sinensis Lam. & 0,34 & Ind \\
\hline & Macrosphyra longistyla (DC.)Hiern & 0,03 & Ind \\
\hline Caricaceae (D) & Carica papaya $\mathrm{L}$. & 0.17 & Exo \\
\hline Casuarinaceae (D) & Casuarina equisetifolia Forst. & 0,17 & Exo \\
\hline \multirow[t]{6}{*}{ Combreataceae (D) } & Combretum micranthum G. Don. & 0,14 & Ind \\
\hline & Guiera senegalensis J.F. Gmel. & 0,07 & Ind \\
\hline & Quisqualis indica $\mathrm{L}$. & 0,14 & Exo \\
\hline & Terminalia catappa $\mathrm{L}$. & 0,07 & Exo \\
\hline & Terminalia macroptera Guill.et Perr. & 0,03 & Ind \\
\hline & Terminalia mantaly Perr. & 0,28 & Exo \\
\hline \multirow[t]{2}{*}{ Cycadaceae (Gymnos) } & Cycas circinalis $\mathrm{L}$. & 0,14 & Exo \\
\hline & Cycas rumphii Miq. & 0,07 & Exo \\
\hline \multirow[t]{13}{*}{ Euphorbiaceae (D) } & Acalypha hispida Burm. & 0.07 & Exo \\
\hline & Acalypha wilkesiana Mull.Arg. & 0.10 & Exo \\
\hline & Codiaeum variegatum (L.) Blume & 0.03 & Exo \\
\hline & Euphorbia lactea Haw. & 0.03 & Ind \\
\hline & Euphorbia tirucalli L. & 0.03 & Ind \\
\hline & Jatropha curcas L. & 0,17 & Exo \\
\hline & Jatropha gossypiifolia L. & 0,03 & Exo \\
\hline & Jatropha integerrima Jacq. & 0,28 & Exo \\
\hline & Jatropha multifida L. & 0,03 & Exo \\
\hline & Macaranga heterophylla Müll.Arg. & 0,07 & Ind \\
\hline & Mallotus oppositifolius (Gers.) Mull.Arg. & 0,07 & Ind \\
\hline & Manihot esculenta Crantz & 0,14 & Exo \\
\hline & Phyllanthus nivosus Smith. & 0,03 & Exo \\
\hline \multirow[t]{5}{*}{ Caesalpinioidea } & Bauhinia purpurea L. & 0.03 & Exo \\
\hline & Bauhinia reticulata DC. & 0.21 & Ind \\
\hline & Caesalpinia pulcherrima (L.) Sw. & 0,24 & Exo \\
\hline & Cassia alata $\mathrm{L}$. & 0.03 & Exo \\
\hline & Cassia siamea Lam. & 0,38 & Exo \\
\hline
\end{tabular}




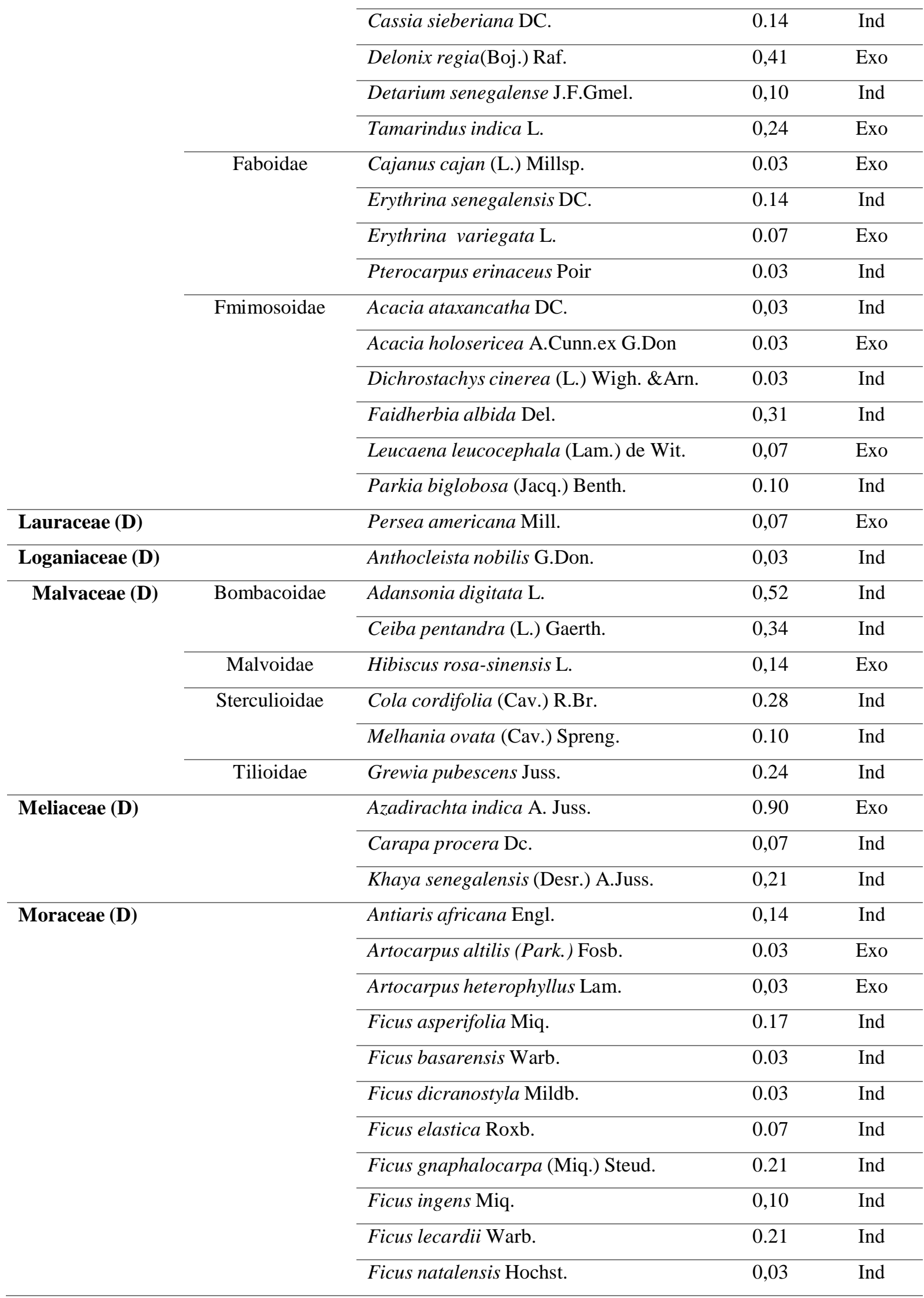




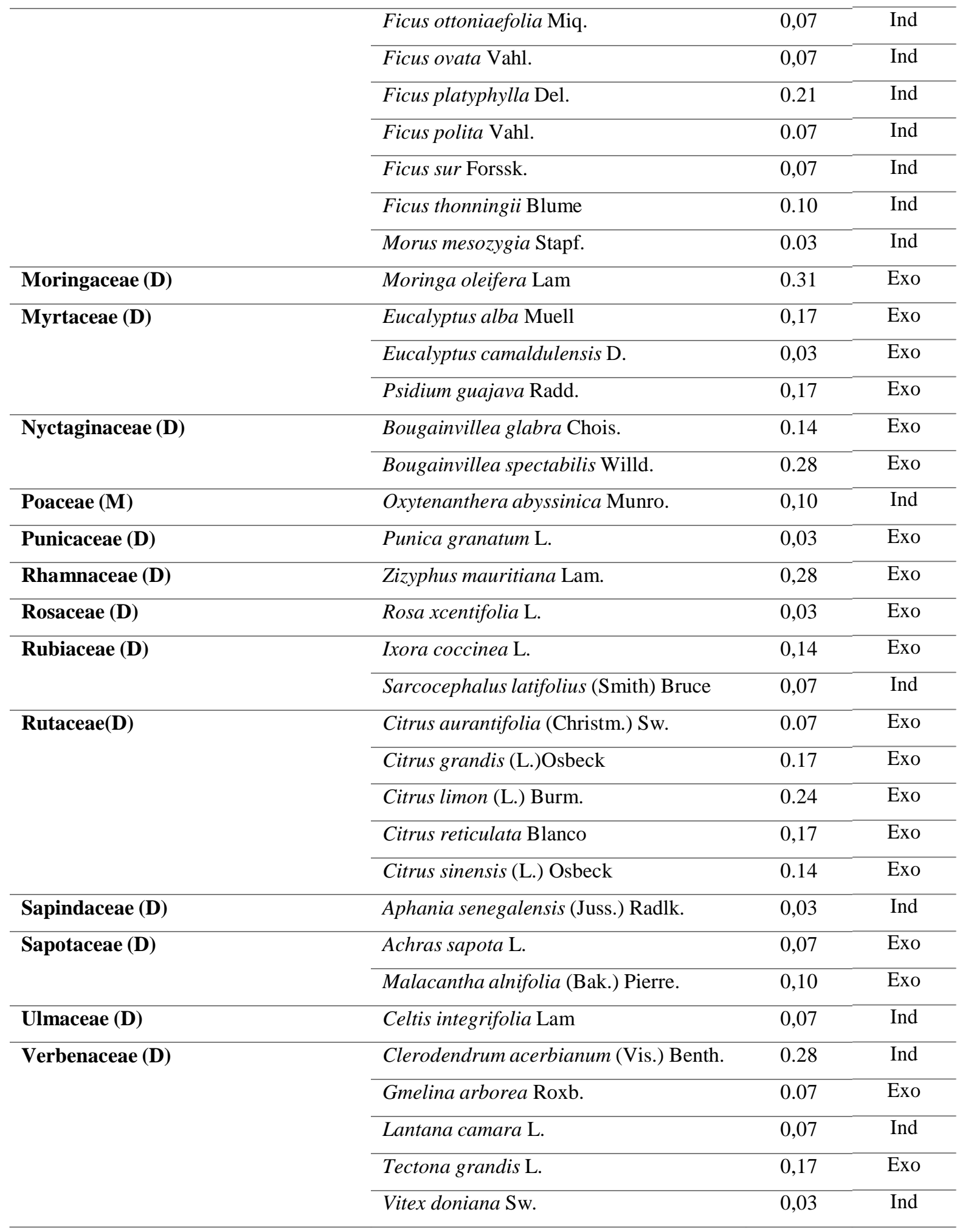


Tableau 2 : Structure taxonomique de la flore.

\begin{tabular}{clcccccc}
\hline \multirow{2}{*}{ Groupes } & \multicolumn{2}{c}{ Familles } & \multicolumn{2}{c}{ Genres } & \multicolumn{2}{c}{ Espèces } \\
\cline { 3 - 8 } ANGIOSPERMES & Monocotylédones & $\mathbf{N}$ & $\mathbf{\%}$ & $\mathbf{N}$ & $\mathbf{\%}$ & $\mathbf{N}$ & $\mathbf{\%}$ \\
\cline { 2 - 8 } & Dicotylédones & 2 & 6,25 & 7 & 7,37 & 7 & 5,3 \\
\cline { 2 - 8 } GYMNOSPERMES & 29 & 90,62 & 87 & 91,58 & 114 & 86,36 \\
\hline \multicolumn{2}{c}{ TOTAL } & 1 & 3,13 & 1 & 1,05 & 2 & 2,11 \\
\hline
\end{tabular}

Tableau 3 : Liste des familles des espèces inventoriées par ordre d'importance.

\begin{tabular}{|c|c|c|c|}
\hline $\mathbf{N}^{\circ}$ & FAMILLES & Nombre d'espèces & $\%$ Famille \\
\hline 1 & Fabaceae (D) & 19 & 14,39 \\
\hline 2 & Moraceae (D) & 18 & 13,64 \\
\hline 3 & Euphorbiaceae (D) & 13 & 9,85 \\
\hline 4 & Apocynaceae (D) & 11 & 8,33 \\
\hline 5 & Combretaceae (D) & 6 & 4,55 \\
\hline 6 & Arecaceae (M) & 6 & 4,55 \\
\hline 7 & Malvaceae (D) & 6 & 4,55 \\
\hline 8 & Verbenaceae (D) & 5 & 3,79 \\
\hline 9 & Rubiaceae (D) & 5 & 3,79 \\
\hline 10 & Bignonaceae (D) & 4 & 3,03 \\
\hline 11 & Araliaceae (D) & 4 & 3,03 \\
\hline 12 & Myrtaceae (D) & 3 & 2,27 \\
\hline 13 & Meliaceae (D) & 3 & 2,27 \\
\hline 14 & Boraginaceae (D) & 3 & 2,27 \\
\hline 15 & Annonaceae (D) & 3 & 2,27 \\
\hline 16 & Sapotaceae (D) & 2 & 1,52 \\
\hline 17 & Rubiaceae (D) & 2 & 1,52 \\
\hline 18 & Nyctaginaceae (D) & 2 & 1,52 \\
\hline 19 & Cycadaceae (G) & 2 & 1,52 \\
\hline 20 & Anacardiaceae (D) & 2 & 1.52 \\
\hline 21 & Agavaceae (D) & 2 & 1,52 \\
\hline 22 & Ulmaceae (D) & 1 & 0,76 \\
\hline 23 & Sapindaceae (D) & 1 & 0,76 \\
\hline 24 & Rosaceae (D) & 1 & 0,76 \\
\hline 25 & Rhamnaceae (D) & 1 & 0,76 \\
\hline 26 & Punicaceae (D) & 1 & 0,76 \\
\hline 27 & Poaceae (M) & 1 & 0,76 \\
\hline 28 & Moringaceae (D) & 1 & 0,76 \\
\hline 29 & Loganiaceae (D) & 1 & 0,76 \\
\hline 30 & Lauraceae (D) & 1 & 0,76 \\
\hline 31 & Casuarinaceae (D) & 1 & 0,76 \\
\hline \multirow[t]{2}{*}{32} & Caricaceae (D) & 1 & 0,76 \\
\hline & & 132 & 100,00 \\
\hline
\end{tabular}




\section{DISCUSSION}

\section{Flore}

\section{Composition floristique}

L'étude a permis de recenser 132 espèces réparties dans 95 genres, relevant de 32 familles. Comparée à la flore vasculaire du Sénégal (Ba, 2001), la flore de la commune de Ziguinchor présente des proportions plus ou moins différentes entre Dicotylédones et Monocotylédones de l'ensemble de la flore du pays. En effet, les Dicotylédones de la ville de Ziguinchor représentent 90,62\% du cortège floristique alors qu'au niveau national ils représentent $\quad 70,3 \%$. Quant aux Monocotylédones, elles sont très faiblement représentées $(6,25 \%)$ par rapport à leur proportion nationale $(18,7 \%)$. Par ailleurs, la flore de la ville de Ziguinchor est aussi plus riche que celle des terres jachères de l'Est du Sénégal et de la Casamance. En fait, les résultats d'une étude récente de Ka et al. (2017) ont montré que la flore de cette dernière est riche de 109 espèces. La structure taxonomique est cependant plus ou moins semblable à celle des systèmes agropastoraux de la communauté rurale de Mlomp (Bassene et al., 2014) à 40 km au sud de Ziguinchor et aussi celle de la. La flore inventoriée dans la ville de Ziguinchor est plus riche que celle de quelques villes africaines étudiées telles que Brazzaville qui compte 43 espèces appartenant à 33 genres et 20 familles dans les sites étudiés, plus 28 espèces autres dans des reliques de forêts situées aux alentours de la ville (N'zala, 2003), Lomé, où on a recensé 93 espèces appartenant à 79 genres et 47 familles (Polorigni et al, 2014); Nouakchott et Ouagadougou présentant des proportions semblables (Dutrève et al, 1998). Certaines espèces de la ville sont communes aux forêts communautaires du Sine-Saloum dans le Centre-Ouest du Sénégal (Charahabil et al, 2013). Il s'agit entre autres des Combretaceae qui continuent une colonisation vers le sud du pays (Guiera senegalensis et Combretum micrantum), et de Grewia pubescens, Leptadenia hastata, et Faidherbia albida qui sont des espèces rustiques adaptées à tous les écosystèmes de la zone tropicale sèche. La nouvelle édition de la flore de Berhaut (1967) a été enrichie de quelques récoltes de la région naturelle de la Casamance, mais l'absence de quelques espèces assez bien représentées dans ce territoire, mais absentes dans cette édition montre qu'il reste encore du travail à faire dans cette zone. C'est le cas de Markhamia tomentosa (Bignonaceae) et de Cordia sebestena (Boraginaceae), une espèce introduite.

\section{Statut écologique}

L'analyse de l'origine des espèces (endogènes ou exotiques) montre que $53 \%$ des arbres et arbustes de la ville sont introduits contre $47 \%$ d'espèces endogènes. Les dix (10) espèces exotiques les plus fréquentes sont respectivement Azadirachta indica, Mangifera indica, Delonix regia, Cassia siamea, Anacardium occidentale, Cocos nucifera, Moringa oleifera, Terminalia mantaly, Jatropha integerrima et Bougainvillea spectabilis. Ces plantes sont utilisées comme essences ornementales, mais aussi dans l'alimentation humaine. Contrairement aux 10 espèces exotiques qui sont faiblement représentées. Il s'agit de Phyllanthus nivosus. Bauhinia purpurea, Cassia alata, Cajanus cajan, Acacia holosericea, Artocarpus altilis, Artocarpus heterophyllus, Eucalyptus camaldulensis, Punica granatum, et Rosa xcentifolia. Par opposition au premier groupe, ces espèces ne sont pas souvent utilisés comme plantes d'avenus, ni comme taxon d'ombres et rarement dans l'alimentation. En effet, le jacquier, l'arbre à pain (A. heterophyllus et A.altilis et le poids d'angole [Cajanus cajan], bien que très consommées dans d'autres pays ne figurent pas dans les habitudes alimentaires des habitants de la ville.

\section{Typologie des habitats et de la végétation Aménagement}

La ville de Ziguinchor s'est développée sur le fleuve Casamance dans le cadre global de l'environnement naturel amphibie des «rivières du sud ». À l'heure actuelle, malgré une certaine horizontalité générale des terrains, la topographie de la ville fait distinguer deux types de paysages :

— «Les terres basses», qui sont des dépôts marins et fluviomarins du quaternaire 
récent, qui s'élèvent jusqu'à 4 ou $5 \mathrm{~m}$ d'altitude, le contact avec le plateau étant plus marqué par la différence de végétation que par le dénivelé topographique [Ville de Ziguinchor 2016]. Il s'agit de la partie Ouest et à Est de la ville, environnant le quartier Escale, site d'origine de la ville. Dans cette zone s'étendait un paysage de «tanne» et de mangrove, mais en grande partie aménagée en rizières qui a longtemps empêché toute extension linéaire de Ziguinchor le long du fleuve.

-«Les plateaux », constitués essentiellement du plateau de Peyrissac et celui de Néma qui retombent sur les cuvettes plus loin à Djibélor d'une part et à Tilène et Kandialang d'autre part. Sur ces plateaux, le faciès des dépôts du continental terminal n'est pas gréseux, mais sablo -argileux et marneux, avec une faible dose ferralitique qui donne cette couleur rouge caractéristique de la terre et des maisons en banco dans les quartiers de plateaux. Dans cette zone, en plus de la subsistance de quelques fromagers originels, en observe un couvert arboré dominé par des manguiers. C'est cette organisation qui permet de faire une analyse approximative de l'organisation issue de l'Analyse Factorielle de Correspondance. Suivant l'axe F1 on retrouve en abscisses positives les espaces végétalisés, localisés essentiellement dans «Les terres basses». Ils sont constitués essentiellement d'une «nouvelle flore» introduite dans les jardins, et les espaces publics. En abscisse négative, ce sont les EVU localisés dans «Les plateaux ». Ils sont essentiellement constitués d'une flore forestière spontanée composée d'espèce du genre Ficus, Anthocleista, Malacantha, Erythrina, Pterocarpus. Cet axe représente un gradient de topographie suivi par une action progressive de l'homme. Ce même gradient topographique, associé au réseau hydrographique de la ville est essentiellement influencé par le fleuve Casamance. L'axe F2 permet, quant à lui, d'isoler en extrémité positive des espèces au statut endogènes et rares inféodées essentiellement aux écosystèmes guinéens. [Ficus. basarensis, F. ovata, $F$. ottoniaefolia, $F$. platyphyla, $F$. leucardii], Detarium. senegalensis, Cola. cordifolia, Ceiba. pentandra, Cassia. sieberiana, Elaeis guinneensis). On retrouve l'essentiel de ces espèces dans les cimetières, des lieux de cultes (fétiches) et dans l'enceinte des camps des sapeurs-pompiers et des militaires. Ces endroits présentant une forte concentration de ces grands arbres sont des zones où la coupe du bois est interdite pour éviter que les personnes aperçoivent ce qui se fait à l'intérieur de ces lieux. Parmi ces activités, il y a l'enterrement des morts qui est considéré sacré pour certaines animistes Diola. Pour ces derniers, les femmes et les garçons non-initiés ne doivent pas voir comment on enterre un mort. Aussi, dans ces lieux de cultes, sous certaines plantes comme Ceiba Pentandra, Cola cordifolia et le genre Ficus sont implantés des fétiches. Les camps militaires sont aussi des endroits où l'accès est réglementé et où les arbres servent des barrières pour empêcher la vue de loin de l'intérieur du camp. À cela, s'ajoute que dans ces camps les bordures de la voirie sont plantées de grands arbres. L'ensemble de ces éléments justifient le rôle que jouent ces zones sacrées et/ou l'accès est difficile aux populations dans la conservation in situ de la biodiversité.

\section{Conclusion}

Ce travail a permis de faire une caractérisation quantitative de la flore ligneuse de la ville de Ziguinchor. Il ressort de cette étude que cette flore est fortement diversifiée avec 132 espèces réunies dans 95 genres et 32 familles, ce qui n'est pas très commun dans nos villes africaines. Cette richesse floristique indique qu'il existe encore un grand réservoir de biodiversité dans cette ville et plus particulièrement dans les zones sacrées et les domaines protégés. Les résultats montrent aussi des proportions comparables entre les espèces endogènes et exogènes. L'hétérogénéité de cette végétation ligneuse témoigne d'une diversité du patron habitat dans la ville. Ces habitats vont des endroits aménagés par l'homme pour son confort urbain aux reliques de forêts relativement bien conservées. Ce travail d'inventaire et d'analyse de la flore devrait être poursuivi dans toutes les 
villes du pays en y intégrant la nature chorologique des espèces et leur importance pour les populations afin de mieux comprendre l'organisation de ces EVU et mieux justifier leur maintien dans les villes.

\section{CONFLIT D'INTÉRÊTS}

Les auteurs déclarent, à l'unanimité, qu'il n'y a aucun conflit d'intérêts qui serait liée au présent article.

\section{CONTRIBUTIONS DES AUTEURS}

MMC: élaboration des outils méthodologique, participation aux inventaires et rédacteur principal ; BC: identification des spécimens d'herbier, traitement et analyse des données floristiques; $\mathrm{BH}$ : inventaire de la flore, traitement des données et rédaction; $\mathrm{SN}$ : cartographie; MD: superviseur de l'équipe.

\section{REFERENCES}

Amontcha AAM, Lougbegnon T, Tente B, Djego J, Sinsin BA. 2015. Aménagements urbains et dégradation de la phytodiversité dans la Commune d'Abomey-Calavi (Sud-Bénin). Journal of Applied Biosciences, 91: 8519 8528.

DOI:

http://dx.doi.org/10.4314/jab.v91i1.9

ANSD. 2011. Sénégal et ICF International.

Enquête Démographique et de Santé à Indicateurs Multiples au Sénégal (EDSMICS) 2010-2011. Calverton, Maryland, USA : ANSD et ICF International. P.520.

ANSD. 2015. Sénégal: Enquête Démographique et de Santé Continue (EDS - Continue). P.270.

Arnberger A, Eder R. 2012. The influence of green space on community attachment of urban and suburban residents. Urban Forestry \& Urban Greening, 11(1): 4149.

DOI:

https://doi.org/10.1016/j.ufug.2011.11.00 3

Arnould P, Le Lay YF, Dodane C, Méliani I, 2011. La nature en ville : l'importable biodiversité. Géographie, économie \& Sociéte, 1 (13): 45-68. DOI : https://halshs.archivesouvertes.fr/halshs-00862457

Ba AT, Noba K. 2001. Flore et biodiversité végétale au Sénégal. Science et Changements Planétaires/Sécheresse, 12(3) : 149-55.

Bassene C, Mbaye MS, Camara AA, Kane A, Gueye M, Sylla SN, Sambou B, Noba K. 2014. La flore des systèmes agropastoraux de la Basse Casamance (Sénégal) : cas de la communauté rurale de Mlomp. International Journal of Biological and Chemical Sciences, 8(5): 2258-2273.

DOI: http://dx.doi.org/10.4314/ijbcs.v8i5.28

Berhaut J. 1967. Flore du Sénégal. (2éme Edn). Clairafrique : Dakar; P.485.

Berhaut J. 1971-1991. Flore Illustrée du Sénégal. Éd. Gouvernement du Sénégal, MRD /DEF. Vol. 1, Acanthacées à Avicenniacées, 628p; vol. 2, Balonophoracées à Composées, 696 $\mathrm{p}$; vol. 3, Connoracées à Euphorbiacées, $634 \mathrm{p}$; vol. 4, Ficoïdes à Légumineuses, $626 \mathrm{p}$; vol. 5, Léégumineuses à papilionacées, $658 \mathrm{p}$; vol. 6, Lynacées à Nymphéacées, 636 p.

Botineau M. 2009. Classification APG III. Guide des plantes à fruits charnus comestibles et toxiques. 295p.

Charahabil MM, Diallo A, Ngom D, Diop

B, Akpo LE. 2013. Importance des Combretaceae dans des forêts communautaires de la zone soudanosahélienne au Sénégal. Science et Changements Planétaires/Sécheresse, 24(1): 39-47.

Cornelis J, Hermy M. 2004. Biodiversity relationships in urban and suburban parks in Flanders. Landscape and Urban Planning: 285-401.

DOI:

https://doi.org/10.1016/j.landurbplan.200 3.10 .038

CSE. 2011. Adaptation aux impacts du changement climatique quelles stratégies d'échanges et de partage de l'information scientifique. P.404.

Dasylva M. 2013. Agriculture urbaine sous pluie et sécurité alimentaire dans la 
commune de Ziguinchor. Mémoire de Master. UASZ/Département d'agroforesterie, Ziguinchor. P.51.

Dasylva M, Ndour N, Ndiaye O, Samba B. 2017. Analyse de la flore, de la végétation ligneuse et des fonctions des vallées en zone péri-urbaine post-conflit (Ziguinchor, Sénégal). International Journal of Biological and Chemical Sciences, 11(1): 360-377. DOI: http://dx.doi.org/10.4314/ijbcs.v11i1.28

Dutrève B, Pinatel M, Besse F. 1998.

Foresterie urbaine. Place de l'arbre urbain dans deux capitales ouest-africaines : Nouakchott et Ouagadougou. Le Flamboyant, 47 : 18-25. DOI : http://catalogue-

bibliotheques.cirad.fr/cgi-bin/koha/opacdetail.pl?biblionumber $=91051$

Huck JF. 1980. Manuel : Botanique forestière et arboretum E.A.T.F. École Des Agents Techniques Des Eaux et forêts, P.84.

Ka SL, Mbaye MS, Gueye M, Bamba B, Ly MO, Diouf N, Noba K. 2017.

Systematic composition, life forms and chorology of fallow lands in Eastern Senegal and Casamance, Senegal. International Journal of Biological and Chemical Sciences, 11(6): 2573-2586. DOI:

http://dx.doi.org/10.4314/ijbcs.v11i6.2

Laille P, Provendier D, Colson F, Salanié J.

2013. Les bienfaits du végétal en ville: étude des travaux scientifiques et méthode d'analyse. Plante \& Cité, Angers, P.31.

MCA-S. 2013. Plan d'action de réinstallation (par). Rapport final. P.234.

MEPN. 1997. Elaboration de la monographie nationale sur la diversité biologique au Sénégal, P.82.

Montoroi JP. 1993. Les sols et agriculture dans le domaine estuarien de Basse Casamance. Département eaux continentales. ORSTOM. ${ }^{\circ} 38.545$ Cote B. P.9.

Mugnier J. 2008. Nouvelle Flore illustrée du Sénégal et des régions voisines. Agroservices.fr, France Google Scholar.
N'Zala D. 2003. L'arbre en ville, étude de foresterie urbaine à Brazzaville, Congo. FAO 2003.

Noba K. 2002. La flore adventice dans le sud du Bassin arachidier (Sénégal) : structure, dynamique et impact sur la production du mil et de l'arachide. Thèse de doctorat d'Etat. UCAD/Sénégal. P.141.

Nomel GJR, Vroh BTA, Gone Bi ZB, Adou Yao CY, N'Guessan KE. 2017. Caractéristiques floristiques et structurales des espaces aménagés au niveau des échangeurs de la ville D'Abidjan (Côte d'Ivoire). International Journal of Biosciences, 9(3).

Polorigni B, Radji R, Kokou K. 2014. Perceptions, tendances et préférences en foresterie urbaine: cas de la ville de Lomé au Togo. European Scientific Journal, 10(5) : 261-277.

Randriambanona HA. 2008. Successions écologiques dans les plantations de Pinus, d'Acacia et dans les forêts naturelles de la région nord-ouest du corridor de Fianarantsoa (Madagascar). Thèse de doctorat en sciences de la vie option: biologie et écologie végétales spécialité : écologie végétale. UCAD. P.137.

Salah R. 1999. La foresterie urbaine et périurbaine en Afrique. Une étude de cas sur le sahel (Dakar, Niamey, Nouakchott et Ouagadougou. Archives de documents de la FAO, P.26. DOI : http://www.fao.org/DOCREP/005/X399 4F/X3994F01.htm

Sané T, Dièye EHB, Descroix L. 2017. Un littoral en mouvement : diversité, dynamiques et lutations des territoires frontaliers du sud- ouest du Sénégal et du nord-ouest de la Guinée-Bissau Montreuil (FRA); Paris (FRA); Ziguinchor: GRDR; IRD; UASZ, P.133. http://www.documentation.ird.fr/hor/fdi: 010071812

Sarr RS, Mbaye MS. 2007. La flore adventice des cultures d'oignons dans la zone périurbaine de Dakar [Niayes] Sénégal. Webbia, 62(2): 205-216. 
Saümel I, Kowarik I, Butenschön S. 2010.

Green traces from past to future: the interplay of culture and ecological processes in European historical parks. Acta Horticul., 881 : 933-938. DOI : https://doi.org/10.17660/ActaHortic.201 0.881 .156

Savard JPL, Clergeau P, Mennechez G. 2000. Biodiversity concepts and urban ecosystems. Landscape and Urban Planning: 131-142. DOI: https://doi.org/10.1016/S01692046(00)000372

Tavin A, Leseur A. 2016. Végétaliser la ville : pour quels bénéfices, avec quels financements, suivis et gouvernances des projets? L'apport d'exemples européens et nord-américains. Institute For Climate Economic. P.32.

Vanden Berghen C. 1982. La végétation des sables de Djibonker, en Basse Casamance
[Sénégal méridional]. Bull. Jard. Bot. Nat. Belg., 52: 211-224. http://www.jstor.org/stable/3668056 Vanden Berghen C. 1998. Irradiations de la flore sahélienne en Basse Casamance [Sénégal]. In : Atelier sur flore, végétation et biodiversité au Sahel, Bâ AT, Madsen JE, Sambou B (eds). AAU Report, 39: 151-161.

Ville de Ziguinchor. 2016. Évolution urbaine de Ziguinchor. http://villedeziguinchor.org/index.php/fr/ component/content/article/8-... 24/07/2016 13 h 52.

Vroh BTA, Tiebre MS, N'guessan KE. 2014. Diversité végétale urbaine et estimation du stock de carbone : cas de la commune du Plateau Abidjan, Côte d'Ivoire. Afrique SCIENCE 10(3): $329-340$. DOI:

http://www.afriquescience.info/ 